\title{
1 From computing transition probabilities to word recognition in 2 sleeping neonates, a two-step neural tale
}

3 Ana Flóa ${ }^{*}$, Lucas Benjamin ${ }^{\mathrm{a}}$, Marie Palu ${ }^{\mathrm{a}}$, and Ghislaine Dehaene-Lambertz ${ }^{\mathrm{a}}$

$4 \quad{ }^{a}$ Cognitive Neuroimaging Unit, CNRS ERL 9003, INSERM U992, CEA, Université Paris-

5 Saclay, NeuroSpin center, 91191 Gif/Yvette, France.

6 * Corresponding autor: Ana Fló.

$7 \quad$ Email: ana.flo@cea.fr

8 Keywords

9 Neonates, Statistical Learning, Language acquisition Neural Entrainment.

10 Abstract

11 Extracting statistical regularities from the environment is a primary learning mechanism, 12 which might support language acquisition. While it is known that infants are sensitive to 13 transition probabilities between syllables in continuous speech, the format of the encoded 14 representation remains unknown. Here we used electrophysiology to investigate how 31 15 full-term neonates process an artificial language build by the random concatenation of four 16 pseudo-words and which information they retain. We used neural entrainment as a marker 17 of the regularities the brain is tracking in the stream during learning. Then, we compared 18 the evoked-related potentials (ERP) to different triplets to further explore the format of the 19 information kept in memory. After only two minutes of familiarization with the artificial 20 language, we observed significant neural entrainment at the word rate over left temporal 21 electrodes compared to a random stream, demonstrating that sleeping neonates 22 automatically and rapidly extracted the word pattern. ERPs significantly differed between 23 triplets starting or not with the correct first syllable in the test phase, but no difference was associated with later violations in transition probabilities, revealing a change in the representation format between segmentation and memory processes. If the transition probabilities were used to segment the stream, the retained representation relied on

27 syllables' ordinal position, but still without a complete representation of the words at this

28 age. Our results revealed a two-step learning strategy, probably involving different brain 29 regions. 


\section{1. Introduction}

2 From before birth, infants demonstrate learning capacities. During the last weeks of 3 gestation, they learned some prosodic features of their native language (Mehler et al., 1988)

4 and their mother's voice (DeCasper \& Fifer, 1980), as the taste of the amniotic liquid

5 (Marlier et al., 1998), and rapidly learn to recognize their mother's face after birth

6 (Bushneil et al., 1989). Neonates also quickly adapt to repeated sensory information. For

7 example, after a few minutes of familiarization with a word, they notice when it changed

8 (Benavides-Varela et al., 2012; Benavides-Varela \& Mehler, 2011), and similar short-term

9 memories have been described for previously seen faces (Pascalis et al., 1995, p. 994).

10 Despite these undeniable learning and memory capacities, very little is known about the

11 underlying mechanisms, the information neonates are sensitive to, and the format of the

12 representation in which information is memorized.

13 Here we focused on a primary yet indispensable fast learning mechanism: statistical

14 learning. Statistical learning refers to the capacity to detect regularities in the input.

15 Abundant literature (Saffran \& Kirkham, 2018) shows that this mechanism is common

16 across domains (visual, auditory) (Bulf et al., 2011; Fiser \& Aslin, 2002; Kirkham et al.,

17 2002; Saffran et al., 1996, 1999), species (primates, rodents) (Hauser et al., 2001; Toro \&

18 Trobalón, 2005), and extends to different levels of stimulus/scene complexity. Concerning

19 language acquisition, statistical learning has been proposed as a critical mechanism to

20 explain how infants might discover linguistic regularities. For example, it might serve to

21 identify word candidates based on frequently co-occurring syllables (Saffran et al., 1996),

22 to discover phonotactic and acoustic patterns (Friederici et al., 2007; Jusczyk et al., 1999),

23 and to detect morphological and syntactic regularities (Shi et al., 1999).

24 Experimental evidence supporting the role of statistical learning in language acquisition

25 has been mainly obtained in word segmentation tasks from an artificial speech stream in

26 which acoustic cues have been removed. In a seminal study (Saffran et al., 1996, p. 996),

27 8-month-old infants were first exposed to 3 minutes of an artificial speech (thereafter called

28 Structured stream) constituted by four randomly concatenated tri-syllabic pseudo-words,

29 with the drops of transition probabilities (TPs) between syllables as the only cue to word 
1 boundaries. Within a pseudo-word, the first two syllables predict the following syllable

2 (TP equal to 1), while the last syllable could be followed by any other pseudo-words (TP

3 equal to $1 / 3$ ). When test triplets were then played in isolation, infants' looking pattern

4 differed between the pseudo-words (i.e., Words: both TPs in the triplet equal 1) and triplets

5 straddling a TP drop (i.e., Part-words: one TP equal 1 and the other equal 1/3). This result,

6 uncovered that infants are sensitive to the statistical relations between syllables, yet, it

7 remains unknown what they exactly learn.

8 It is commonly assumed that infants segment the stream into words that are memorized

9 and subsequently recognized when presented in isolation. However, two other hypotheses

10 can also explain the results. Infants may compute the transitional probabilities matrix

11 between all syllables through synaptic plasticity and Hebbian learning (Endress \& Johnson,

12 2021) without segmenting the stream (Benjamin et al., in prep.). The different association

13 strength between syllables in Words and Part-Words could support the difference between

14 these conditions. Alternatively, infants may segment the stream using the drop of

15 transitional probabilities at the end of the Words but only memorize the syllable following

16 the drop. Indeed, because this syllable being less predictable during the stream, it might

17 induce surprise, a powerful learning factor for infants (Stahl \& Feigenson, 2015). The three

18 hypotheses are not dissociable in the existing studies since they all result in differential

19 responses to Words and Part-words. Nevertheless, each explanation relies on different

20 mechanisms in terms of computational complexity and neural bases.

21 A crucial difference between encoding the TPs matrix and segmenting the stream into

22 Words is that memory constraints for sequence encoding may enter into play. When

23 encoding a sequence of items, each item is associated with the close items (i.e., TPs) and

24 its ordinal position within the sequence (Henson, 1998). Indeed, Dehaene et al. (Dehaene

25 et al., 2015) proposed a taxonomy of five levels along which a sequence can be encoded:

26 from (1) TPs between elements, passing by (2) chunking (grouping of elements in a unit),

27 and (3) ordinal knowledge (the elements have an ordered position in the unit), until more

28 abstract encoding based on (4) rules and (5) nested structures. In a very recent study in 23

29 adult patients with implanted electrodes who listened to a structured stream containing

30 Words, the first stages of this taxonomy were explored using representational similarity 
1 analyses. The authors reported a complex picture in which different brain regions hosted

2 different representations (Henin et al., 2021). Some electrodes, located in the superior

3 temporal gyrus and the pars opercularis and motor cortex, were responding to TPs

4 encoding. Others, located in the inferior frontal gyrus, anterior temporal lobe, and posterior

5 superior temporal sulcus, were sensitive to ordinal position (first vs. second vs. third

6 syllable). Finally, in the hippocampus, electrodes were sensitive to Words (chunks). Given

7 the complex maturational calendar of the different brain structures, particularly the slow

8 maturation of the hippocampus (Lavenex \& Banta Lavenex, 2013) and frontal areas

9 (Lebenberg et al., 2019), the generalization of these results to young infants might be

10 inadequate. Furthermore, attention is notably limited at a young age, especially in neonates

11 who sleep most of the time. Thus, we may wonder whether passive exposure might be

12 sufficient or whether some of these computations, such as representing syllables' ordinal

13 position and active prediction of the next item, might not be observed during sleep. In other

14 words, our goal was to study which levels of this taxonomy newborns possess to support

15 language acquisition.

16 Three previous studies have shown that neonates are at least sensitive to the first level, TPs

17 encoding. During a long familiarization with an artificial stream of syllables (15 $\mathrm{mn})$

18 (Teinonen et al., 2009) and of tones (9 mn) (Kudo et al., 2011), a different event-related

19 responses emerged to the first syllables/tones of the Words. However, this result may

20 reflect either the response to a local prediction error (i.e., TPs), or to changes in the syllable

21 frequencies as the medial and last syllable were repeated from time to time, or to truly

22 individual triplets. A third study using Near-Infrared Spectroscopy (NIRS) showed a

23 differential BOLD response to Words and Part-words following a 3.5 minutes

24 familiarization with the structure stream (Fló et al., 2019). While adding that neonates can

25 remember the extracted information for a few minutes, it leaves pending the information

26 they retained that triggered the differential response. We, therefore, propose here to further

27 investigate the study of statistical learning in neonates using high-density EEG (128

28 electrodes) in a paradigm based on three minutes of exposure to a structured stream,

29 followed by the presentation of isolated triplets in a test phase (Fig 1). Our goal was double,

30 first to target learning cues during the stream exposure thanks to neural entrainment, and 
1 second to characterize the format of the learned representation by presenting four different

2 types of triplets in isolation.

3 Thanks to its temporal sensitivity, EEG allows monitoring learning, even in non-

4 participating subjects, such as sleeping neonates. In particular, in this paradigm, where

5 syllables have a fixed duration, the auditory response induced by the regular presentation

6 can be captured as entrainment at the frequency of stimulation ( $\mathrm{f}=1 /$ syllable duration).

7 Crucially, this steady-state response is not limited to low-level features like syllable onset

8 but can reflect any regular pattern the brain is tracking. Thus, if the listener detects the 3-

9 syllabic pattern embedded in the stream, entrainment should also be observed at the triplet

10 frequency (1/3 of the syllabic rate) (Batterink \& Choi, 2021; Benjamin et al., 2021; Buiatti

11 et al., 2009; Kabdebon et al., 2015). Here we quantified the entrained neural responses at

12 the syllabic and Word rates measuring an enhanced Power and Inter Trial Coherence (ITC)

13 during the presentation of the Structured stream, a Random stream (random concatenation

14 of the syllables), and Resting-state (i.e., no stimulation). We expected a similar entrainment

15 at the syllabic rate for both streams relative to resting-state, but an increased activity at the

16 word rate during the Structured stream.

17 However, neural entrainment at the word frequency can result from two different 18 processes, similarly to differential ERPs to syllables during the stream (Kudo et al., 2011;

19 Teinonen et al., 2009). Either the neonates have detected a drop in TPs, or they recognize

20 the triplets re-occurrence. To test what they learned and memorized, we compared the

21 ERPs to isolated triplets in a post-learning phase (Fig 1B). We build four types of triplets

22 to disentangle different hypotheses on the encoding format of the memorized pattern. We

23 contrasted: (1) triplets respecting, or not, TPs between syllables, and (2) triplets violating,

24 or not, the ordinal position of the first syllable. Therefore, we presented the classical

25 conditions: Words $\left(A_{i} B_{i} C_{i}\right)$ corresponding to the pseudo-words present in the stream, and

26 Part-words $\left(B_{i} C_{i} A_{k}\right)$ corresponding to triplets straddling a TP drop. Note that Part-words

27 also have an incorrect first syllable. To these common conditions, we added two other

28 conditions: Edge-words and Non-words. Edge-words $\left(A_{i} B_{i} C_{k}\right)$ were triplets in which the

29 last syllable between two Words was exchanged; thus, they retained the ordinal position of

30 the syllables, but they were never presented in the stream (last TP equaled zero). Non- 
1 words $\left(B_{i} C_{i} A_{i}\right)$ were triplets in which the first syllable appeared in the last position; thus,

2 all syllables belonged to the same Word, but the ordinal position was incorrect, and the

3 triplet was never heard (last TP equaled zero).

A

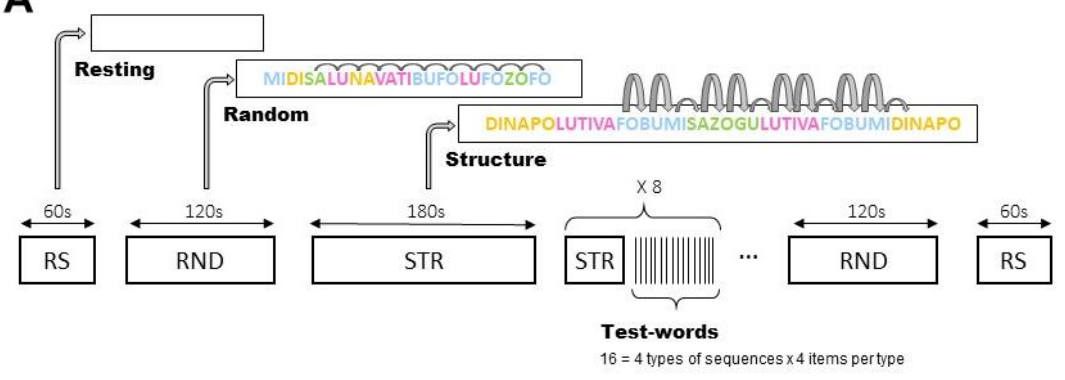

B

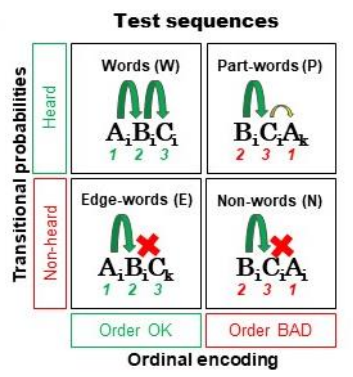

5 Figure 1. (A) Experimental protocol. A long Structured stream (180 s) was followed by 8 test short

6 familiarizations (30 s) and test blocks, where 16 triplets were presented in isolation (ISI 2-2.5 s).

7 Resting state and a Random stream were sandwiching the entire experiment to control for the effect

8 of time, and notably infant vigilance, on the EEG recordings. (B) Possible types of test words. Test

9 words could have violations in the TPs between the second and third syllables, in the ordinal

10 position of the syllables, or both.

11 If neonates segment the stream and encode ordinal information or at least the first syllable

12 of a word, we expected an early differential response between $A B x$ (Words and Edge-

13 Words) and $B C x$ triplets (Part-Word and Non-Words). Note that any difference before the

14 third syllable can only be due to the encoding of the first syllable $-A_{i} B_{i}$ and $B_{i} C_{i}$ had both

15 TPs equal to one. By contrast, if the response to the isolated triplets only depends on the

16 adherence to the statistical structure of the Structured stream, the ERPs between never

17 heard triplets (Edge-words and Non-words) and those present in the stream (Words and

18 Part-words) should differ from the third syllable. For the sake of completeness, we also

19 considered that memory encoding following segmentation might be sensitive to the

20 temporal proximity of the elements belonging to the same chunk as a community structure,

21 predicting that Non-Words $\left(B_{i} C_{i} A_{i}\right)$ are closer to Words $\left(A_{i} B_{i} C_{i}\right)$ than Part-Words $\left(B_{i} C_{i} A_{k}\right)$.

22 Additionally, we tested 32 adult participants in a behavioral online experiment analog to

23 the infant task. After familiarization with the structured stream, participants had to rate

24 their familiarity with the test triplets. Because the stimuli (duration of the Structured 
1 streams and number of tests words) were the same as in the neonates' study, this experiment

2 provides a reference point of what mature and expert participants encode and memorize.

3 To summarize, simple TP learning should result in a difference between triplets present or

4 not in the stream (Words+Part-words vs. Edge-words+Non-words). Segmentation should

5 be revealed by neural entrainment at the word rate during the Structured stream and a

6 difference between $A B x$ and $B C x$ sequences. The granularity of the encoding can be further

7 investigated by comparing Words vs. Edge-Words and Non-words vs. Part-Words.

\section{2. Materials and Methods}

\section{2.1. Participants}

10 Participants were healthy-full-term neonates, with normal pregnancy and birth (GA > 38

11 weeks, Apgar scores $\geq 7 / 8$ in the $1 / 5$ minute, birthweight $>2.5 \mathrm{Kg}$, cranial perimeter $\geq 33.0$

$12 \mathrm{~cm}$ ), tested at the Port Royal Maternity (AP-HP), in Paris, France. Parents provided

13 informed consent. 31 participants who provided enough data without motion artifacts were

14 included (10 females; 1 to 4 days old; mean GA: 40.2 weeks; mean weight: 3475 g). Seven

15 other infants were excluded from the analysis (3 due to excessive hair or cradle cap, 2 due

16 to excessive motion artifacts, and 2 because the parents decided to interrupt the

17 experiment).

\subsection{Stimuli}

19 The stimuli were synthesized using the fr4 French female voice of the MBROLA diphone 20 database (Dutoit et al., 1996). Syllables had a consonant-vowel structure. Each phone had 21 a duration of $125 \mathrm{~ms}$ and a constant pitch of $200 \mathrm{~Hz}$. The streams were continuous with co22 articulation and no pauses, and they were ramped up and down during the first and last $5 \mathrm{~s}$ 23 to avoid the start and end might serve as perceptual anchors. The structured streams 24 consisted of a semi-random concatenation of the four tri-syllabic pseudo-words. Pseudo25 words were concatenated with the only restrictions that the same word could not appear 26 twice in a row, and the same two words could not repeatedly alternate more than two times

27 (i.e., the sequence $W_{k} W_{j} W_{k} W_{j}$, where $W_{k}$ and $W_{j}$ are two words, was forbidden). The 28 learning stream lasted 180 seconds, each word appearing 60 times and each of the 12 
1 possible part-words 18 to 21 times; the average TPs between words was $0.332(\mathrm{SD}=0.017$,

2 range 0.310 to 0.361 ). The eight short structured streams lasted 30 seconds each, each word

3 appearing $80(8 \times 10)$ times and each of the 12 possible part-words between 24 and 28 times;

4 the average transitional probability between words was 0.325 ( $\mathrm{SD}=0.012$, range 0.308 to

5 0.345). The pseudo-words were created to avoid that specific phonetic features could help

6 to segment the stream. Additionally, three different structured streams (lists A, B, and C,

7 corresponding to three sets of pseudo-words) were used by modifying how the syllables

8 were combined to form the Words (Table 1). Participants were randomly assigned and

9 balanced between lists.

10 Table 1. Triplets for each condition (Words, Edge-words, Part-words, and Non-words) used for 11 each of the three lists.

\begin{tabular}{ccccc}
\hline List & $\mathbf{A}_{\mathbf{i}} \mathbf{B}_{\mathbf{i}} \mathbf{C}_{\mathbf{i}}$ & $\mathbf{A}_{\mathbf{i}} \mathbf{B}_{\mathbf{i}} \mathbf{C}_{\mathbf{k}}$ & $\mathbf{B}_{\mathbf{i}} \mathbf{C}_{\mathbf{i}} \mathbf{A}_{\mathbf{k}}$ & $\mathbf{B}_{\mathbf{i}} \mathbf{C}_{\mathbf{i}} \mathbf{A}_{\mathbf{i}}$ \\
\hline & dinapo & dinava & napolu & napodi \\
A & lutiva & lutimi & tivafo & tivalu \\
& fobumi & fobugu & bumisa & bumifo \\
& sazogu & sazopo & zogudi & zogusa \\
\hline & napolu & napofo & poluti & poluna \\
B & tivafo & tivasa & vafobu & vafoti \\
& bumisa & bumidi & misazo & misabu \\
& zogudi & zogulu & gudina & gudizo \\
\hline & poluti & polubu & lutiva & lutipo \\
& vafobu & vafozo & fobumi & fobuma \\
C & misazo & misana & sazogu & sazomi \\
& gudina & guditi & dinapo & dinagu \\
\hline
\end{tabular}

13 The random were built a semi-random concatenation of the same 12 syllables, with the 14 only restriction that the same syllable could not appear twice in a row and that two syllables

15 could not alternate more than two times (i.e., the sequence $S_{k} S_{j} S_{k} S_{j}$, where $S_{k}$ and $S_{j}$ are two

16 syllables, was forbidden). Streams lasted 120 seconds each, and each syllable appeared 480

17 times in each of them.

18 Test words were tri-syllabic triplets presented in isolation.

\section{2.3. Procedure and data acquisition}


1 Scalp electrophysiological activity was recorded using a 128-electrode net (Electrical

2 Geodesics, Inc.) referred to the vertex with a sampling frequency of $250 \mathrm{~Hz}$. Neonates were

3 tested in a soundproof booth while sleeping or during quiet rest. The study involved: (1)

460 s of resting-state; (2) $120 \mathrm{~s}$ of a random stream; (3) $180 \mathrm{~s}$ of a structured stream (4) 8

5 series of a $30 \mathrm{~s}$ of structured streams followed by 16 test sequences (ISI 2-2.5s); (5) $120 \mathrm{~s}$

6 of a random stream; (6) $60 \mathrm{~s}$ of resting state. The random streams and resting-state were

7 presented before and after the learning and test parts to avoid sensing entrainment

8 differences due to time in the experiment (i.e., a change in the vigilance state).

\section{2.4. Data pre-processing}

10 Data were band-pass filter $0.1-40 \mathrm{~Hz}$ and pre-processed using custom MATLAB scripts

11 based on the EEGLAB toolbox 2021.0 (Delorme \& Makeig, 2004), according to the

12 APICE pre-processing pipeline (Fló et al., 2021). The following steps were applied: (1)

13 Data was band-pass filter 0.1-40 Hz. (2) Artifacts were identified based on voltage

14 amplitude, variance, first derivative, and running average using adaptive thresholds. (3)

15 Time samples were defined as artifacts if more than $30 \%$ of the channels presented

16 artifacts. (4) Electrodes were spatially interpolated using spherical splines for samples that

17 were not marked as bad.

\section{$18 \quad 2.5$. Neural entrainment}

19 The pre-processed data were resampled to $300 \mathrm{~Hz}$ to achieve an integer number of samples

20 per triplet (225 samples in $0.75 \mathrm{~s}$ ) and further high-pass filtered at $0.2 \mathrm{~Hz}$. Then, data was

21 segmented from the beginning of each phase into $0.75 \mathrm{~s}$ long segments. Segments

22 containing samples with artifacts were rejected. On average we retained $74 \%$ of the data

23 during Resting (SD 17, range [31, 100]), $84 \%$ of the data during the Random (SD, 11, [47,

24 100]), and $87 \%$ of the data during the Structured (SD 7, range [71, 100]). Subjects who

25 did not provide at least 6 segments per condition were not included.

26 Neural entrainment per condition. The $0.75 \mathrm{~s}$ epochs belonging to the same condition were

27 reshaped into non-overlapping epochs of $7.5 \mathrm{~s}$ (10 triplets, 30 syllables), retaining the

28 chronological order; thus, the timing of the steady state response. Data were referenced 
1 average and normalized by dividing by the standard deviation within an epoch. DSS, a

2 technique based on spatial filters designed to remove stimulus-unrelated activity (de

3 Cheveigné \& Simon, 2008), was applied, and the first 30 components of the first PCA and

4 the first 6 of the DSS filter were retained (the pattern of results did not differ if DSS was

5 not used). Then, data were converted to the frequency domain using the Fast Fourier

6 Transform (FFT) algorithm, and the power and ITC were estimated for each electrode

7 during each condition (Resting-state, Random, Structured). Then, the SNR relative to the

8 twelve adjacent frequency bins ( six of each side corresponding to $0.8 \mathrm{~Hz}$ ) was estimated.

9 The power was computed as the power spectrum of the average response across trials.

10 Then, the noise level was estimated at each frequency by assuming a power-law fit on the

11 adjacent frequency bins $\log \left(P_{\text {estimate }}(f)\right)=a+b * \log (f)$. The SNR for the power was $\operatorname{SNR}(f)$

$12=\left(\log (P(f))-\operatorname{mean}\left(P_{\text {noise }}(f)\right)\right) / \operatorname{std}\left(P_{\text {noise }}(f)\right)$, where $P_{\text {noise }}(f)=\log \left(P_{\text {estimate }}(f)\right)-\log (P)$. The

13 ITC was computed as $\operatorname{ITC}(f)=\frac{1}{N}\left|\sum_{i=1}^{N} e^{i \varphi(f, i)}\right|$, where $N$ is the number of trials and $\varphi(f, i)$

14 is the phase at frequency $f$ and trial $i$. The ITC ranges from 0 to 1 (i.e., completely

15 desynchronized activity to perfectly phased locked activity). The SNR was also estimated

16 using the twelve adjacent frequency bins (six of each side corresponding to $0.8 \mathrm{~Hz}$ ) as

$17 \operatorname{SNR}(f)=\left(\operatorname{ITC}(f)\right.$-mean $\left.\left(I T C_{\text {noise }}(f)\right)\right) /$ std $\left(I T C_{\text {noise }}(f)\right)$, where $I T C_{\text {noise }}(f)$ is the ITC over the

18 adjacent frequency bins.

19 For statistical analysis, the SNR was compared at the syllabic rate $(4 \mathrm{~Hz})$ and word rate

$20(1.33 \mathrm{~Hz})$ against zero (null hypothesis) using a one-tail t-test. P-values were corrected

21 across electrodes by FDR.

22 Neural entrainment time course. The 0.75 s epochs were concatenated chronologically (1

23 minute of RS, 2 minutes of Random, 3 minutes of long Structured stream, 4 minutes of

24 short Structure blocks, 2 minutes of Random, and 1 minute of RS). The same analysis than

25 above was performed in sliding time windows of 2 minutes with a $1.5 \mathrm{~s}$ step.

\subsection{ERPs to test words}

27 The pre-processed data were filtered between 0.5 and $20 \mathrm{~Hz}$, epoched between [-1.50, 3.25]

$28 \mathrm{~s}$ from the onset of the triplets. Epochs containing samples identified as artifacts were

29 rejected. On average we retained 24.8 trials for the Word condition (SD 3.6, range [17, 
$131]$ ), 24.5 for the Edge-word condition (SD 3.6, range [16, 30]), 24.7 for the Part-word 2 condition (SD 4.0, range [16, 30]), and 24.5 for the Non-word condition (SD 4.0, range

$3[14,30])$. Subjects who did not provide at least 12 trials per condition were excluded. Data

4 were reference averaged, normalized by dividing by its standard deviation, and baseline

5 corrected by subtracting the average over the interval between $2.25 \mathrm{~s}$ from the onset of the

6 previous word and the corresponding word. Trials were averaged by condition, and two

7 contrasts were studied: (1) $A B x$ (Words and Edge-words) vs. $B C x$ (Part-words and Non-

8 words) triplets; (2) triplets with heard transitions (Words and Part-words) vs. un-heard

9 transitions (Edge-words and Non-words). The responses were compared using non-

10 parametric cluster-based permutation analysis (Oostenveld et al., 2011) in two time

11 windows: (1) $[0,0.5]$ s to detect early effects only attributable to the encoding of the first

12 syllable, and (2) $[0.5,2.75] \mathrm{s}$ to detect effects related to a TPs violation or the triplets'

13 offset. A t-statistic with an alpha threshold of 0.05 was used for clustering; neighbor

14 electrodes had a maximum distance of $3 \mathrm{~cm}$ (4.2 neighbors per channel on average);

15 clusters had a minimum size of two, and 5,000 permutations were run to estimate the

16 significance level. The quantification of the effect along test blocks was performed by

17 computing the average difference between $A B x$ and $B C x$ conditions over the clusters. Data

18 points were included for subjects and blocks when at least 3 out of 8 trials in both

19 conditions were included.

\subsection{Adult behavioral experiment}

2133 French speaking adults were tested in an online experiment analogous to the infant study

22 through the Prolific platform and received monetary compensation for their participation.

23 The same stimuli as in the infant experiment were used. Participants first heard 3 minutes

24 of familiarization with the Structured stream. Then, they completed eight sessions of re-

25 familiarization and testing. Each re-familiarization lasted $30 \mathrm{~s}$, and in each test session, all

2616 possible test words were presented. Subjects were said they should try to identify the

27 words from an invented language. During the test phase, subjects had to report after each

28 test word their familiarity with it. Subjects responded by using the cursor to click on the

29 screen on a scale from 1 to 6 . One participant was excluded because (s)he always responded

30 with a score of 1 or 2 . Subjects were randomly assigned to one of the three lists. 

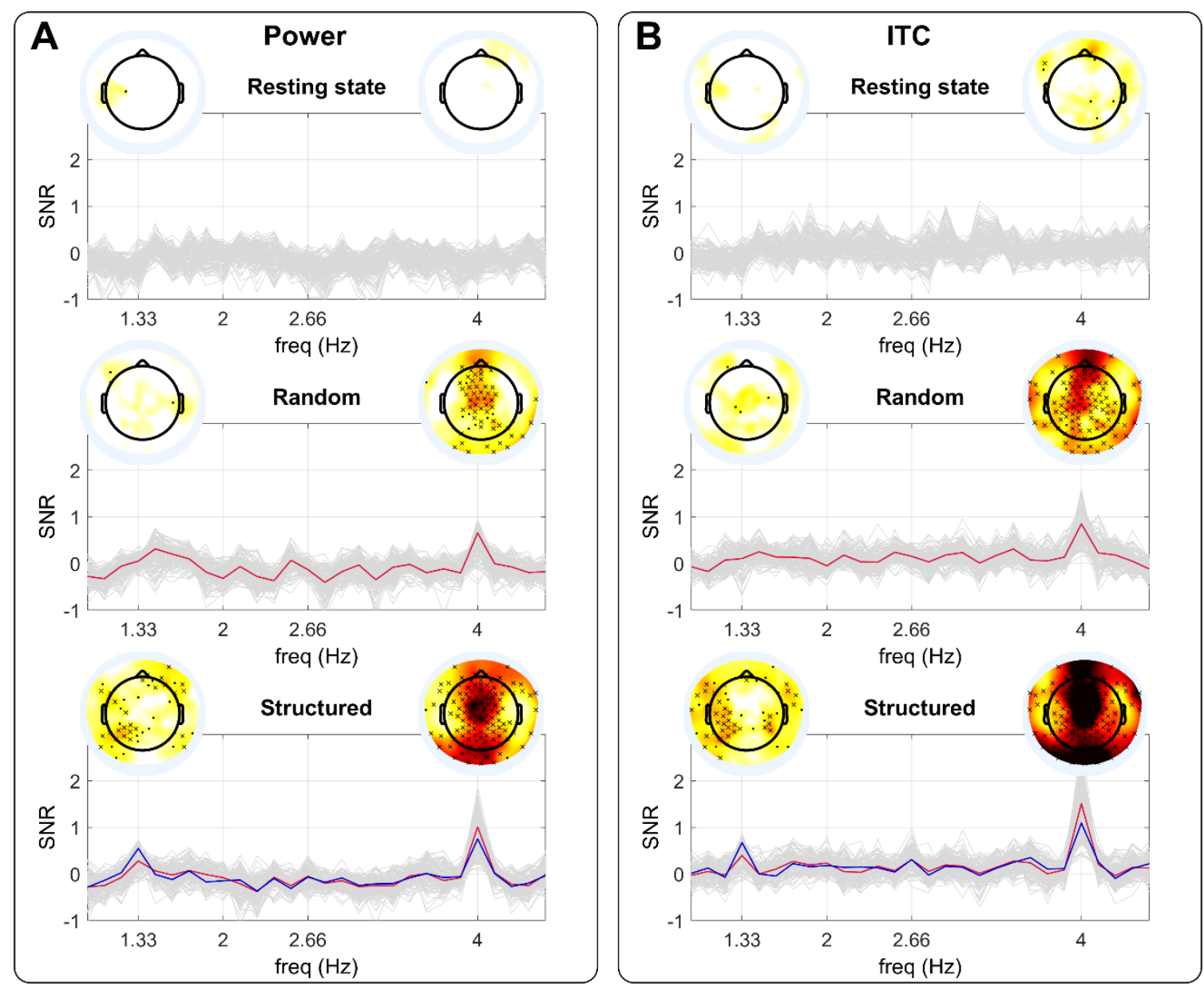

2 Figure 2. Neural entrainment during Resting-state, Random streams, and Structured streams. (A)

3 SNR for the power. In light gray, the entrainment for all electrodes. In red, the mean over the

4 electrodes showing significant entrainment $(p<0.05$, one-sided t-test, FDR corrected) at the

5 syllabic rate $(4 \mathrm{~Hz})$. In blue, the mean over the electrodes showing significant entrainment $(p<$

60.05 , one-sided t-test, FDR corrected) at the word rate $(1.33 \mathrm{~Hz})$. The topographies represent the

7 entrainment in the electrodes space at the word rate $(1.33 \mathrm{~Hz})$ and at the syllabic rate $(4 \mathrm{~Hz})$.

8 Asterisks indicate the electrodes showing enhanced neural activity (cross: $p<0.05$, one-sided t-

9 test, FDR corrected; dot: $p<0.05$, one-sided t-test, without FDR correction). (B) Same based on

10 ITC.

\section{3. Results}

\section{3.1. Neural markers of learning in neonates: familiarization phase}

13 During Resting-state, as expected, no entertainment was seen either at the syllabic (4 Hz)

14 or word $(1.33 \mathrm{~Hz})$ rates. As expected, for Random streams, we observed enhanced activity 
1 at the syllabic rate for many central-frontal and posterior electrodes $(p<0.05$, FDR

2 corrected) and no enhanced activity at the word rate. During the Structured streams, we

3 observed a similar enhanced oscillatory activity at the syllabic rate and significant neural

4 entrainment at the word rate mainly over left temporal electrodes $(p<0.05$, FDR corrected)

5 (Figure 2).

6 By comparing the entrainment with a 1-way-ANOVA with condition as within-subjects

7 factor (Fig. $3 \mathrm{~A}$ and B), similar results were obtained for power and ITC. A main effect of

8 condition was observed at the syllabic rate (power: $F(2,58)=21.8, p=8.6 x 10^{-08}$, ITC:

$9 F(2,58)=21.8, p=8.7 \times 10^{-8}$, driven by a lower power/ITC during Resting than Random

10 (power: $p=0.0021$, ITC: $p=0.0085)$ and Structured $\left(p=8.4 \times 10^{-9}\right.$, ITC: $\left.\mathrm{p}=7.5 \times 10^{-9}\right)$, and

11 lower power/ITC during Random than Structured (power: $p=0.0075$, ITC: $p=0.0017$ ).

12 At word rate there was a main effect of condition (power: $F(2,58)=10.7, p=0.00018$,

13 ITC: $F(2,58)=8.2, p=0.000706$ ), due to a higher power/ITC during Structured than

14 Resting (power: $p=2.9 \times 10^{-5}$, ITC: $p=0.00038$ ) and Random (power: $p=0.0052$, ITC: $p$

$15=0.013)$. All p-values were Bonferroni corrected for multiple comparisons.

16 To quantify learning through the experiment, we measured entrainment at the syllabic and

17 word rate in sliding time windows of 2 minutes with a $1.5 \mathrm{~s}$ step by concatenating the data

18 from all conditions. Notice that because the integration window is two minutes long, the

19 entrainment during the first minute of random, for example includes, data from the

20 structured stream. Results show an increase in Power and ITC at the word rate at around 2

21 minutes from the beginning of the structured stream (Figure $3 \mathrm{C}$ and $\mathrm{D}$ ).

\section{3.2. Encoding format in neonates: Post-learning phase}

23 We first looked for ERPs components related to ordinal position violations by comparing $24 A B x$ (Words and Edge-words) vs. $B C x$ triplets (Part-words and Non-words). A non25 parametric cluster-based permutation analysis (Oostenveld et al., 2011) revealed a 26 significant early difference before $500 \mathrm{~ms}$ in a positive frontal cluster $(p=0.0152$, time 27 window $[0,388] \mathrm{ms})$ and in a left-posterior negative cluster $(p=0.0324$, time window [0, 28 308] ms), (Fig. 4 A, B). Given the time window, this effect can only be related to the 29 encoding of the first syllable (i.e., ordinal encoding). A second difference was also 
1 observed after the offset of the triplet, in a frontal-left positive cluster $(p=0.0142$, time

2 window $[788,1600] \mathrm{ms})$, and even a third one later in a frontal cluster $(p=0.002$, time

3 window [1684, 2628] ms) (Fig. 4 C, D).
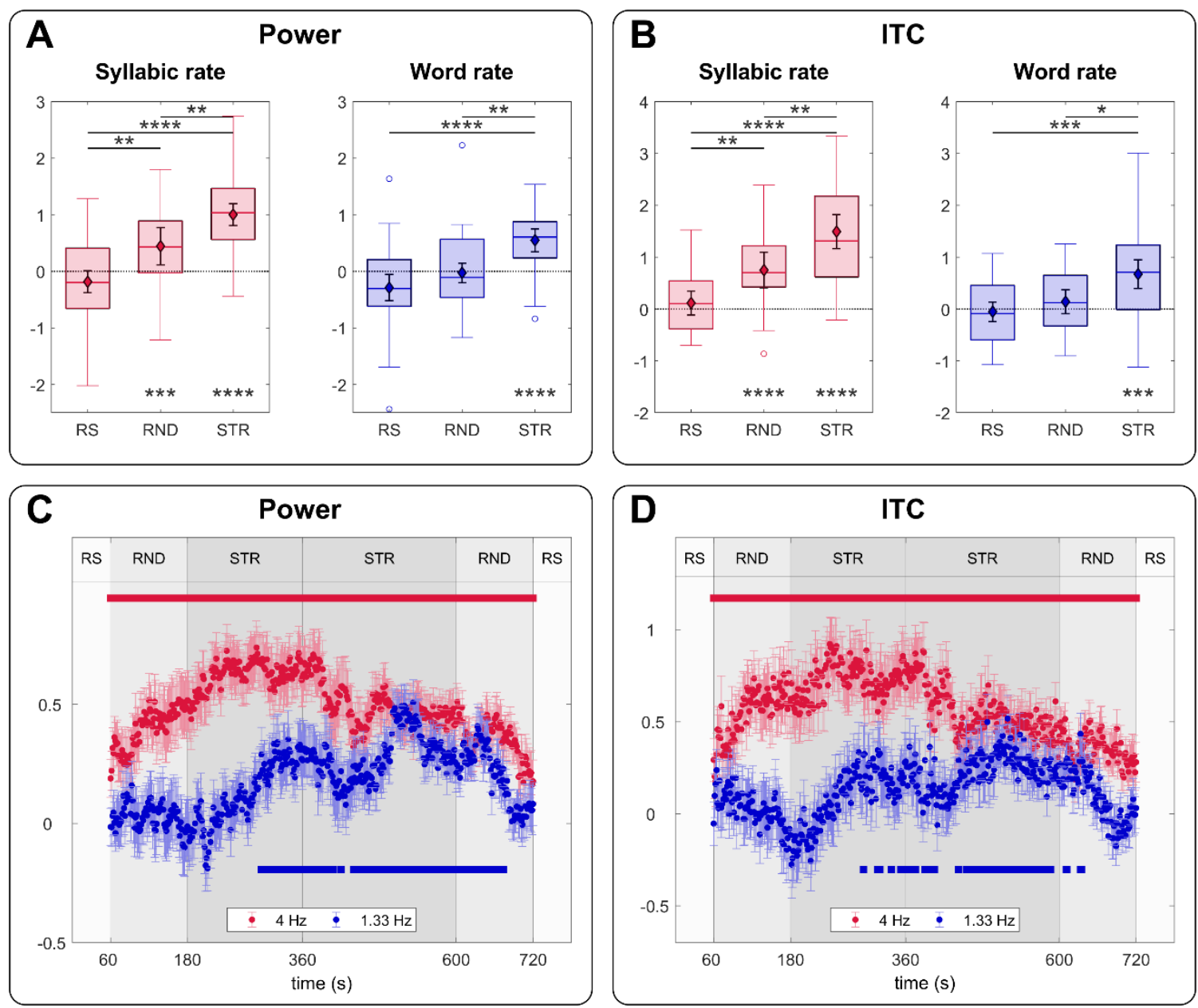

5 Figure 3. (A) SNR for the power at the Syllable and Word rate during the three conditions. Asterisks

6 represent Bonferroni corrected p-values. (B) Same as A based on ITC. (C) Time course of

7 entertainment based on power computed on $120 \mathrm{~s}$ time windows. Error bars represent standard

8 errors. The red line on the top indicates when the power at the Syllabic rate $(4 \mathrm{~Hz})$ was bigger than

9 the null hypothesis 0 ( $p<0.05$, one-sided t-test, corrected by FDR). The blue line on the bottom

10 indicates when the power at the Word rate $(1.33 \mathrm{~Hz})$ was bigger than the null hypothesis $0(p<$

110.05 , one-sided t-test, corrected by FDR). (D) Same as C based on ITC.

12 We then looked for ERPs components related to TPs violations by comparing heard triplets

13 (Words and Part-words) vs. non-heard triplets (Edge-words and Non-words), but we found 
1 no significant difference $(p>0.1)$. No significant differences were detected in the

2 comparisons Words vs. Edge-words, and Part-words vs. Non-words $(p>0.1)$.

3 To ensure that ordinal encoding was present from the beginning of the test phase and was

4 not triggered by hearing isolated triplets, we computed the effect throughout the eight test

5 blocks. Despite fluctuations likely due to the small number of trials, the effect was present

6 from the earliest test blocks (Fig. 4E), suggesting that the encoding of the first syllable in

7 Words had emerged while infants were listening to continuous speech.
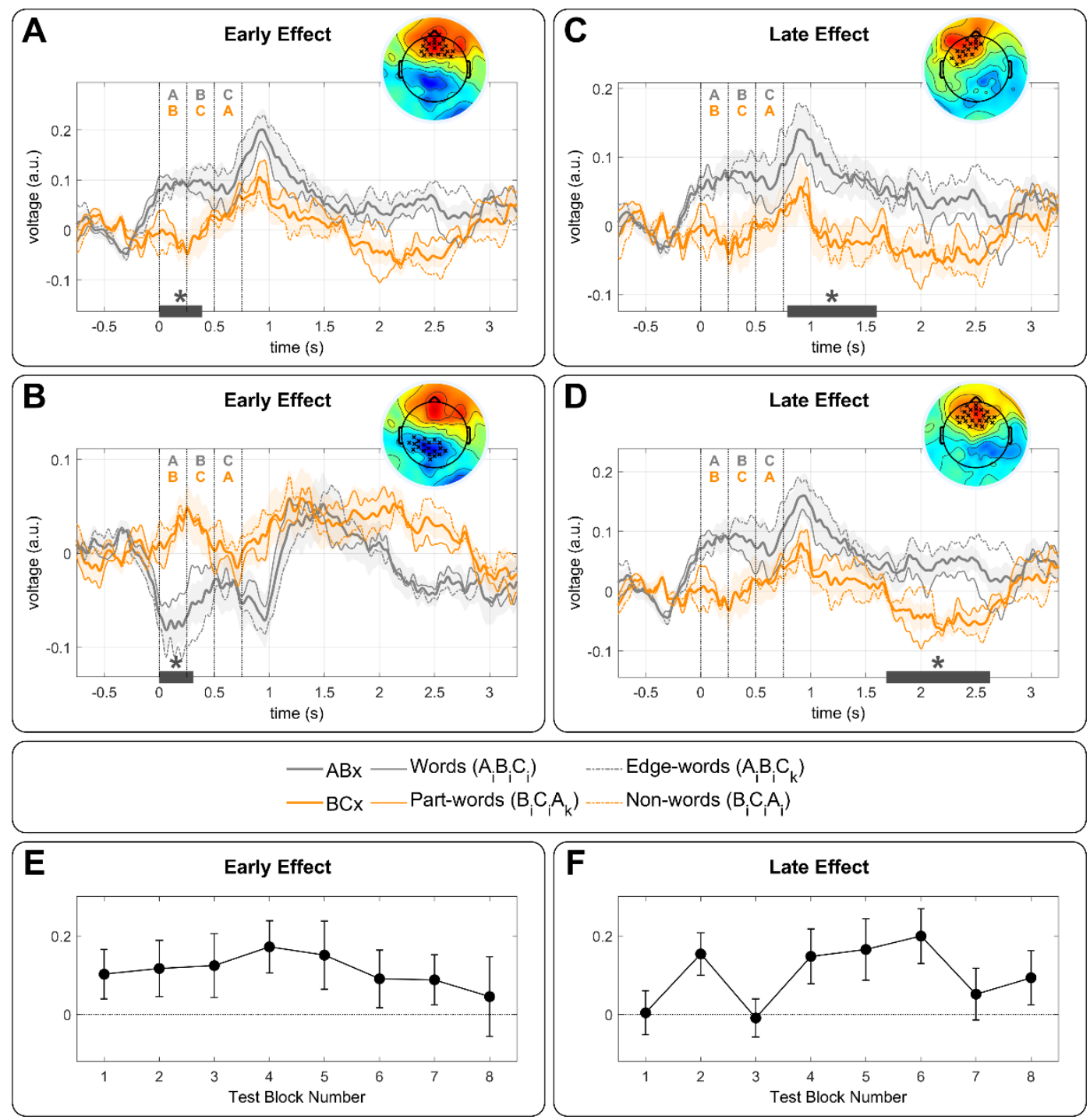

9 Figure 4. Responses to triplets in isolation. (A) Grand-average response to $A B x$ and $B C x$ triplets

10 over the early frontal positive cluster $(p=0.0152)$ obtained from the cluster-based permutation

11 analysis. The thick lines correspond to $A B x$ (gray line) and $B C x$ (orange line) conditions and the 
1 thin lines to the sub-conditions (Words and Edge-Words vs. Part-words and Non-words). Shaded

2 areas correspond to the standard error across neonates. The time zero corresponds to the onset

3 of the test word. The topography shows the difference $A B x-B C x$ during the time window where

4 significant differences were observed (gray line under the plot). (B) Same as A, but for the early

5 negative cluster over left-temporal posterior electrodes $(p=0.0324)$. (C) Same as $A$, but for a late

6 positive cluster over frontal-left electrodes $(p=0.0142)$. (D) Same as A, but for a later positive

7 cluster over frontal electrodes $(p=0.0020)$. (E) Time progression of the ERP early effects $(A$, and

8 B) over the 8 test blocks. (F) Time progression of the average of the two late ERP effects (C, and

9 D) over the 8 test blocks.

\section{$10 \quad 3.3$. Encoding format in adults}

11 Adults rated on a scale their familiarity with the triplets after familiarization with identical

12 streams as neonates (Fig. 5). Results from a linear mixed model using the scoring as

13 dependent variable, the triplet condition as predictor, and subjects as a random factor

14 (Scoring $\sim$ Cnd $+1 \mid$ Sbj) showed a main effect of condition $(F(3,3721)=79.72, p<$

$\left.152.2 \times 10^{-16}\right)$. A post hoc Tukey test revealed that the Words score was higher than each of

16 the other conditions ( $p s<0.0001)$ whereas the Non-words was the lowest, significantly

17 inferior to Part-words $(p<0.0001)$, and to Edge-words $(p=0.0045)$.

\section{4. Discussion}

19 Here, we used a classical speech segmentation task (Saffran et al., 1996) to investigate

20 statistical learning in neonates. While previous studies have shown that infants are sensitive

21 to statistical regularities in speech since birth (Fló et al., 2019; Kudo et al., 2011; Teinonen

22 et al., 2009), it was still unknown what information they tracked and memorized. First, our

23 study revealed that sleeping neonates responded rapidly (within 2 minutes) to the tri-

24 syllabic pattern. Second, when isolated triplets were presented, a differential response was

25 observed from the first syllable, revealing that they expected triplets to start with a specific

26 set of syllables. Third, TP violation did not modulate ERP to triplets. This result indicates

27 a memory representation that no longer depended on TPs, despite TP were used to segment

28 the stream, suggesting a switch to a different format. Finally, these results reveal the

29 accuracy of consonant encoding in newborns that allow them to keep the relationship

30 between 12 syllables and memorize a set of 4 first syllables despite common vowels at 
1 different ordinal word positions. This observation is not trivial given the common

2 assumption that infants are initially limited to the most stable units, such as vowels. For

3 example, Benavides et al. (Benavides-Varela et al., 2012) reported a larger novelty

4 response when changing the vowels of a bi-syllabic word (e.g., lili to lala) compared to a

5 change of consonants (e.g., lili to titi). However, a recent EEG study showed that phonetic

6 features were at the basis of speech perception in 3-month-old pre-babbling infants,

7 opening the possibility of an innate code based on phonetic features for all phonemes,

8 vowels, and consonants (Gennari et al., 2021).

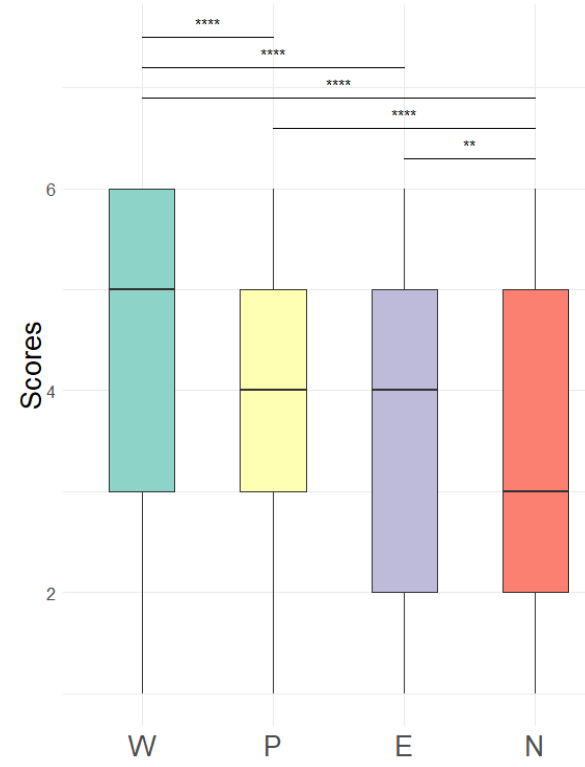

10 Figure 5. Results for the adults' behavioral experiment. The distribution of the scores for all trials 11 and participants are represented per condition.

\section{4.1. Learning based on TPs}

13 The significant increase in power and ITC to word rate in the Structured stream

14 demonstrated that TP computations lead to stream structuring. Learning occurred within 2

15 min of familiarization. This rapid learning is consistent with the length of the stream

16 previously used in behavioral experiments in 8-month-old infants (Saffran et al., 1996) and

17 EEG experiments in adults and 6-month-old infants (Batterink \& Choi, 2021; Benjamin et

18 al., 2021). The concordance of learning rate across ages indicates that statistical learning

19 abilities do not improve markedly with age, a remarkable observation given the significant 
1 maturational changes in auditory/linguistic regions and hippocampus during the first years

2 of life (Adibpour et al., 2020; Leroy et al., 2011).

3 We did not characterize the neonates' sleep stages. However, their general behavior during

4 the recording session (eyes closed, hypotonia), the duration of the experiment, and the lack

5 of task and reward, combined with the short awake periods outside of feeding in the days

6 after birth, certainly did not favor an attentive and focused listening of the auditory input.

7 Neonates' success in extracting the regularities is congruent with adult studies showing

8 neural entrainment at the word rate even when participants are distracted by a primary task

9 (Batterink \& Choi, 2021; Benjamin et al., 2021), revealing the automaticity of TP

10 calculations.

11 In adult experiments, the word rate entrainment is accompanied by decreased syllabic rate

12 entrainment (Batterink \& Choi, 2021). Our results revealed a more complex pattern. The

13 syllabic rate entrainment increased at the beginning of the Structured stream and decreased

14 when word rate entrainment became significant. The initial increase entrainment at the

15 syllabic rate might reflect stronger activation of the language network during the

16 uncovering of the structure compared to random syllable presentation. This hypothesis

17 would be consistent with an adult functional magnetic resonance imaging (fMRI)

18 experiment showing that activity in the left-temporal cortex is modulated by the level of

19 complexity of speech sequences (Pallier et al., 2011). The subsequent decrease might result

20 from top-down inhibition of the syllabic response once the stream has been segmented.

21 While neural entrainment demonstrated that infants were sensitive to the rhythmic structure

22 of the stream, this might result from an automatic error response elicited by the

23 unpredictability of the first syllable (TPs) or by a neural response to tri-syllabic chunks

24 (segmentation). The responses to isolated triplets dissociated these two alternatives.

\section{4.2. Memory representation of the segmented words}

26 ERPs to the isolated triplets revealed the format of the memorized information. ERPs

27 differed from the first syllable between $A B x$ triplets (Words and Edge-Words) and $B C x$

28 triplets (Parts-Words and Non-Words); thus, before any TP violation ( $A B$ and $B C$ 
1 transitions were both equal to 1). Additionally, we observed no specific ERP component

2 after a TPs violation, that is to say, between Words and Edge-Words on one side and Part-

3 Words and Non-Words on the other side. It is important to note that in Non-words, the first

4 syllable was presented at the last position without evoking a particular response (i.e., a

5 difference with Part-Words). The absence of a distinctive response to the first syllable at

6 the wrong position favors the hypothesis that it is not a particular familiarity with this

7 syllable that caused the difference between $A B x$ and $B C x$ triplets, but its position as a first

8 syllable in the triplet.

9 Two approaches have been proposed for flat continuous speech segmentation. From one

10 perspective, the TPs are computed, and the drops in TPs serve as cues to word boundaries

11 (Saffran et al., 1996). From another perspective, recurrent chunks of co-occurring syllables

12 are identified and stored in memory (Perruchet \& Vinter, 1998). Our experiment did not

13 attempt to disentangle these two mechanisms. However, the lack of difference between

14 heard and un-heard triplets revealed that neonates retained neither the full TP matrix nor

15 the entire Words. Instead, they remained limited to the memorization of the first syllable.

16 Meanwhile, adults scored Words as highly familiar, Edge-words as more familiar than

17 Non-words, and finally Edge-Words and Part-words as equally familiar (although Edge-

18 words never appeared in the stream). These results suggest that adults memorized the

19 complete Words, but also that the format of the representation depended on both TPs and

20 ordinal encoding, in agreement with other recent studies (Fló, 2021; Henin et al., 2021).

21 Altogether, our results suggest a multistep process. Segmentation occurred either because 22 the drop in TP produced a prediction error that singularized the non-predicted syllable (i.e.,

23 the first syllables) or because the association of the syllables within words increased their

24 similarity, leading to boundaries at the landscape lower points. In a second step, the

25 segmented triplets are stored in memory, and therefore, any memory constraints might

26 affect the retained representation.

\section{4.3. Word memorization is incomplete in neonates}

28 Neonates are thus memorizing at least the first syllable of the chunk pointing to an ordinal

29 encoding, the third level of complexity in Dehaene et al. taxonomy (Dehaene et al., 2015). 
1 However, they did not distinguish Words $\left(A_{i} B_{i} C_{i}\right)$ and Edge-words $\left(A_{i} B_{i} C_{k}\right)$, suggesting

2 that neonates' words memory was not complete. A limited memory capacity in neonates

3 for middle positions has already been described. A NIRS study showed a better encoding

4 of the syllables at the edges of a six-syllable pseudo word than in intermediate positions

5 (Ferry et al., 2016). Unfortunately, the conditions in that study do not allow disentangling

6 if the effect was due to a better encoding of the first, the last, or both syllables. A memory

7 capacity limited to the first syllable might explain previous neonates studies such as the

8 recognition of bi-syllabic pseudo-words from a new pseudo-word presented two minutes

9 later (Benavides-Varela et al., 2012; Benavides-Varela \& Mehler, 2011) and of words

10 conforming a structured stream (Fló et al., 2019).

11 This memory limitation might be due either to immaturity or to sleep. Sleep is primarily

12 considered as consolidating memories, and while learning is suppressed during deep non-

13 REM stage in adults, implicit word learning is present during REM sleep (Andrillon et al.,

14 2017). Thus, sleep might not represent the limiting factor here, and this question should be

15 further studied.

\subsection{Putative underlying neural networks}

17 While EEG has an excellent temporal resolution, it does not provide accurate spatial

18 resolution and information regarding the activity of brain structures. However, we may

19 speculate from the adults' results and the few brain imaging studies in infants investigating

20 the maturation of the pertinent brain regions. Henin and colleagues (Henin et al., 2021)

21 isolated three main networks in a similar task in epileptic patients that might already be at

22 work in neonates. The superior temporal region, which might be related to local processes

23 involved in TP computations, and two memory structures: the dorsal linguistic pathway

24 supporting verbal working memory, and the hippocampus, recently reported as engaged in

25 sequence learning (Schapiro et al., 2014; Schlichting et al., 2017). Although these two

26 structures have been considered immature in infants, fMRI has revealed that they support

27 cognitive functions in the first trimester. Notably, whereas the superior temporal regions

28 are affected by the immediate repetition of a sentence (Dehaene-Lambertz, 2017),

29 repetition at a longer time-scale of 14 seconds produces activation in the inferior frontal 
1 gyrus in three-month-old infants (Dehaene-Lambertz et al., 2006). Moreover, a NIRS study

2 in sleeping neonates revealed that a correlated activity between left-temporal and left-

3 frontal regions, compatible with activation in the dorsal linguistic pathway, is crucial for

4 word learning (Benavides-Varela et al., 2017). As for the hippocampus, activity has been

5 reported in infants as young as 3-months when performing a visual sequence learning task,

6 with no modulation by infant's age (Ellis et al., 2020). Thus, future work should investigate

7 whether hippocampal circuits considered fundamental to SL, such as the monosynaptic

8 pathway, are involved in such a word-learning task since birth. fMRI in infants might help

9 determine how the network highlighted in adults (Henin et al., 2021) is similarly involved

10 in infants to support the two stages we have isolated the relative role of the hippocampus

11 and the linguistic network.

\section{5. Conclusions}

13 Despite their unquestionable immaturity, neonates reveal sophisticated learning abilities.

14 From drops in TPs, they were able to segment a continuous speech stream and start to

15 encode the first syllables of the chunks. While the present study remains a toy experiment

16 far from the complexity of a real-life environment, it reveals the necessary integration

17 between successive functional processes computed in different neural structures that is at

18 the core of learning in infants.

\section{Acknowledgments}

20 This research has received funding from the European Research Council (ERC) under the

21 European Union's Horizon 2020 research and innovation program (grant agreement No.

22 695710).

\section{Author Contributions}

24 A.F. and G.D.L. designed the research; A.F., L.B. and M.P. performed the research; A.F. 25 analyzed the data; and A.F., L.B., and G.D.-L. wrote the paper

\section{References}


Adibpour, P., Lebenberg, J., Kabdebon, C., Dehaene-Lambertz, G., \& Dubois, J. (2020). Anatomofunctional correlates of auditory development in infancy. Developmental Cognitive Neuroscience, 42, 100752. https://doi.org/10.1016/j.dcn.2019.100752

Andrillon, T., Pressnitzer, D., Léger, D., \& Kouider, S. (2017). Formation and suppression of acoustic memories during human sleep. Nature Communications, 8(1), 179. https://doi.org/10.1038/s41467-017-00071-z

Batterink, L. J., \& Choi, D. (2021). Optimizing steady-state responses to index statistical learning: Response to Benjamin and colleagues. Cortex. https://doi.org/10.1016/j.cortex.2021.06.008

Benavides-Varela, S., Hochmann, J.-R., Macagno, F., Nespor, M., \& Mehler, J. (2012). Newborn's brain activity signals the origin of word memories. Proceedings of the National Academy of Sciences, 109(44), 17908-17913. https://doi.org/10.1073/pnas.1205413109

Benavides-Varela, S., \& Mehler, J. (2011). Memory in the Neonate Brain. PLoS ONE, 6(11), 7.

Benavides-Varela, S., Siugzdaite, R., Gómez, D. M., Macagno, F., Cattarossi, L., \& Mehler, J. (2017). Brain regions and functional interactions supporting early word recognition in the face of input variability. Proceedings of the National Academy of Sciences, 114(29), 75887593. https://doi.org/10.1073/pnas.1617589114

Benjamin, L., Dehaene-Lambertz, G., \& Fló, A. (2021). Remarks on the analysis of steady-state responses: Spurious artifacts introduced by overlapping epochs. Cortex. https://doi.org/10.1016/j.cortex.2021.05.023

Buiatti, M., Peña, M., \& Dehaene-Lambertz, G. (2009). Investigating the neural correlates of continuous speech computation with frequency-tagged neuroelectric responses. NeuroImage, 44(2), 509-519. https://doi.org/10.1016/j.neuroimage.2008.09.015

Bulf, H., Johnson, S. P., \& Valenza, E. (2011). Visual statistical learning in the newborn infant. Cognition, 121(1), 127-132. https://doi.org/10.1016/j.cognition.2011.06.010

Bushneil, I. W. R., Sai, F., \& Mullin, J. T. (1989). Neonatal recognition of the mother's face. British Journal of Developmental Psychology, 7(1), 3-15. https://doi.org/10.1111/j.2044835X.1989.tb00784.x

de Cheveigné, A., \& Simon, J. Z. (2008). Denoising based on spatial filtering. Journal of Neuroscience Methods, 171(2), 331-339. https://doi.org/10.1016/j.jneumeth.2008.03.015

DeCasper, A. J., \& Fifer, W. P. (1980). Of human bonding: Newborns prefer their mothers' voices. Science (New York, N.Y.), 208(4448), 1174-1176. https://doi.org/10.1126/science.7375928 
Dehaene, S., Meyniel, F., Wacongne, C., Wang, L., \& Pallier, C. (2015). The Neural Representation of Sequences: From Transition Probabilities to Algebraic Patterns and Linguistic Trees. Neuron, 88(1), 2-19. https://doi.org/10.1016/j.neuron.2015.09.019

Dehaene-Lambertz, G. (2017). The human infant brain: A neural architecture able to learn language. Psychonomic Bulletin and Review, 24(1), 48-55. https://doi.org/10.3758/s13423-016-1156-9

Dehaene-Lambertz, G., Dehaene, S., Anton, J. L., Campagne, A., Ciuciu, P., Dehaene, G. P., Denghien, I., Jobert, A., LeBihan, D., Sigman, M., Pallier, C., \& Poline, J. B. (2006). Functional segregation of cortical language areas by sentence repetition. Human Brain Mapping, 27(5), 360-371. https://doi.org/10.1002/hbm.20250

Delorme, A., \& Makeig, S. (2004). EEGLAB: An open source toolbox for analysis of single-trial EEG dynamics including independent component analysis. Journal of Neuroscience Methods, 134(1), 9-21. https://doi.org/10.1016/j.jneumeth.2003.10.009

Dutoit, T., Pagel, V., Pierret, N., Bataille, F., \& van der Vrecken, O. (1996). The MBROLA project: Towards a set of high quality speech synthesizers free of use for non commercial purposes. Proceeding of Fourth International Conference on Spoken Language Processing. ICSLP '96, 3, 1393-1396. https://doi.org/10.1109/ICSLP.1996.607874

Ellis, C. T., Skalaban, L. J., Yates, T. S., Bejjanki, V. R., Córdova, N. I., \& Turk-Browne, N. B. (2020). Re-imagining fMRI for awake behaving infants. Nature Communications, 11(1), 4523. https://doi.org/10.1038/s41467-020-18286-y

Endress, A. D., \& Johnson, S. P. (2021). When forgetting fosters learning: A neural network model for statistical learning. 104621. https://doi.org/10.1016/j.cognition.2021.104621

Ferry, A. L., Fló, A., Brusini, P., Cattarossi, L., Macagno, F., Nespor, M., \& Mehler, J. (2016). On the edge of language acquisition: Inherent constraints on encoding multisyllabic sequences in the neonate brain. Developmental Science, 19(3), 488-503. https://doi.org/10.1111/desc.12323

Fiser, J., \& Aslin, R. N. (2002). Statistical learning of new visual feature combinations by infants. Proceedings of the National Academy of Sciences of the United States of America, 99(24), 15822-6. https://doi.org/10.1073/pnas.232472899

Fló, A. (2021). Evidence of ordinal position encoding of sequences extracted from continuous speech. Cognition, 104646. https://doi.org/10.1016/j.cognition.2021.104646

Fló, A., Brusini, P., Macagno, F., Nespor, M., Mehler, J., \& Ferry, A. L. (2019). Newborns are sensitive to multiple cues for word segmentation in continuous speech. Developmental Science, $O(0)$, e12802. https://doi.org/10.1111/desc.12802 
Fló, A., Gennari, G., Benjamin, L., \& Dehaene-Lambertz, G. (2021). Automated Pipeline for Infants Continuous EEG (APICE): A flexible pipeline for developmental studies. BioRxiv, 2021.05.21.445085. https://doi.org/10.1101/2021.05.21.445085

Friederici, A. D., Friedrich, M., \& Christophe, A. (2007). Brain Responses in 4-Month-Old Infants Are Already Language Specific. Current Biology, 17(14), 1208-1211. https://doi.org/10.1016/j.cub.2007.06.011

Gennari, G., Marti, S., Palu, M., Fló, A., \& Dehaene-Lambertz, G. (2021). Orthogonal neural codes for phonetic features in the infant brain. BioRxiv, 2021.03.28.437156. https://doi.org/10.1101/2021.03.28.437156

Hauser, M. D., Newport, E. L., \& Aslin, R. N. (2001). Segmentation of the speech stream in a nonhuman primate: Statistical learning in cotton-top tamarins. Cognition, 78(3), 53-64. https://doi.org/10.1016/S0010-0277(00)00132-3

Henin, S., Turk-Browne, N. B., Friedman, D., Liu, A., Dugan, P., Flinker, A., Doyle, W., Devinsky, O., \& Melloni, L. (2021). Learning hierarchical sequence representations across human cortex and hippocampus. Science Advances, 7(8), eabc4530. https://doi.org/10.1126/sciadv.abc4530

Henson, R. N. A. (1998). Short-Term Memory for Serial Order: The Start-End Model. Cognitive Psychology, 36(2), 73-137. https://doi.org/10.1006/cogp.1998.0685

Jusczyk, P. W., Houston, D. M., \& Newsome, M. (1999). The beginnings of word segmentation in english-learning infants. Cognitive Psychology, 39(3-4), 159-207. https://doi.org/10.1006/cogp.1999.0716

Kabdebon, C., Pena, M., Buiatti, M., \& Dehaene-Lambertz, G. (2015). Electrophysiological evidence of statistical learning of long-distance dependencies in 8-month-old preterm and full-term infants. Brain and Language, 148, 25-36. https://doi.org/10.1016/j.bandl.2015.03.005

Kirkham, N. Z., Kirkham, N. Z., Slemmer, J. a, Slemmer, J. a, Johnson, S. P., \& Johnson, S. P. (2002). Visual statistical learning in infancy: Evidence for a domain-general learning mechanism. Cognition, 83, 4-5.

Kudo, N., Nonaka, Y., Mizuno, N., Mizuno, K., \& Okanoya, K. (2011). On-line statistical segmentation of a non-speech auditory stream in neonates as demonstrated by event-related brain potentials. Developmental Science, 14(5), 1100-1106. https://doi.org/10.1111/j.1467-7687.2011.01056.x

Lavenex, P., \& Banta Lavenex, P. (2013). Building hippocampal circuits to learn and remember: Insights into the development of human memory. Behavioural Brain Research, 254, 8-21. https://doi.org/10.1016/j.bbr.2013.02.007 
1 Lebenberg, J., Mangin, J. F., Thirion, B., Poupon, C., Hertz-Pannier, L., Leroy, F., Adibpour, P., Dehaene-Lambertz, G., \& Dubois, J. (2019). Mapping the asynchrony of cortical maturation in the infant brain: A MRI multi-parametric clustering approach. NeuroImage, 185(September 2017), 641-653. https://doi.org/10.1016/j.neuroimage.2018.07.022

Leroy, F., Glasel, H., Dubois, J., Hertz-Pannier, L., Thirion, B., Mangin, J.-F., \& DehaeneLambertz, G. (2011). Early Maturation of the Linguistic Dorsal Pathway in Human Infants. Journal of Neuroscience, 31(4), 1500-1506. https://doi.org/10.1523/JNEUROSCI.414110.2011

Marlier, L., Schaal, B., \& Soussignan, R. (1998). Neonatal Responsiveness to the Odor of Amniotic and Lacteal Fluids: A Test of Perinatal Chemosensory Continuity. Child Development, 69(3), 611-623. https://doi.org/10.1111/j.1467-8624.1998.tb06232.x

Mehler, J., Jusczyk, P. W., Lambertz, G., Halsted, N., Bertoncini, J., \& Amiel-Tison, C. (1988). A precursor of language acquisition in young infants. Cognition, 29(2), 143-178. https://doi.org/10.1016/0010-0277(88)90035-2

Oostenveld, R., Fries, P., Maris, E., \& Schoffelen, J.-M. (2011). FieldTrip: Open Source Software for Advanced Analysis of MEG, EEG, and Invasive Electrophysiological Data. Computational Intelligence and Neuroscience, 2011, 1-9. https://doi.org/10.1155/2011/156869

Pallier, C., Devauchelle, A.-D., \& Dehaene, S. (2011). Cortical representation of the constituent structure of sentences. Proceedings of the National Academy of Sciences of the United States of America, 108(6), 2522-2527. https://doi.org/10.1073/pnas.1018711108

Pascalis, O., de Schonen, S., Morton, J., Deruelle, C., \& Fabre-Grenet, M. (1995). Mother's face recognition by neonates: A replication and an extension. Infant Behavior and Development, 18(1), 79-85. https://doi.org/10.1016/0163-6383(95)90009-8

Perruchet, P., \& Vinter, A. (1998). PARSER: A Model for Word Segmentation. Journal of Memory and Language, 39, 246-263. https://doi.org/10.1006/jmla.1998.2576

Saffran, J. R., Aslin, R. N., \& Newport, E. L. (1996). Statistical learning by 8-month-old infants. (Vol. 274). https://doi.org/10.1126/science.274.5294.1926

Saffran, J. R., Johnson, E. K., Aslin, R. N., \& Newport, E. L. (1999). Statistical learning of tone sequences by human infants and adults. Cognition, 70(1), 27-52. https://doi.org/10.1016/S0010-0277(98)00075-4

Saffran, J. R., \& Kirkham, N. Z. (2018). Infant Statistical Learning. Annual Review of Psychology, 69(1), 181-203. https://doi.org/10.1146/annurev-psych-122216-011805 
1 Schapiro, A. C., Gregory, E., Landau, B., McCloskey, M., \& Turk-Browne, N. B. (2014). The Necessity of the Medial Temporal Lobe for Statistical Learning. Journal of Cognitive Neuroscience, 26(8), 1736-1747. https://doi.org/10.1162/jocn_a_00578

Schlichting, M. L., Guarino, K. F., Schapiro, A. C., Turk-Browne, N. B., \& Preston, A. R. (2017). Hippocampal Structure Predicts Statistical Learning and Associative Inference Abilities during Development. Journal of Cognitive Neuroscience, 29(1), 37-51. https://doi.org/10.1162/jocn_a_01028

Shi, R., Werker, J. F., \& Morgan, J. L. (1999). Newborn infants' sensitivity to perceptual cues to lexical and grammatical words. Cognition, 72(2), B11-B21. https://doi.org/10.1016/S0010-0277(99)00047-5

Stahl, A. E., \& Feigenson, L. (2015). Observing the unexpected enhances infants' learning and exploration. Science, 348(6230), 91-94. https://doi.org/10.1126/science.aaa3799

Teinonen, T., Fellman, V., Näätänen, R., Alku, P., \& Huotilainen, M. (2009). Statistical language learning in neonates revealed by event-related brain potentials. BMC Neuroscience, 10, 21. https://doi.org/10.1186/1471-2202-10-21

Toro, J. M., \& Trobalón, J. B. (2005). Statistical computations over a speech stream in a rodent. Perception \& Psychophysics, 67(5), 867-875. https://doi.org/10.3758/BF03193539 